\title{
Philosophiques
}

\section{La philosophie des sciences après Kuhn}

\section{Robert Nadeau}

Volume 21, numéro 1, printemps 1994

URI : https://id.erudit.org/iderudit/027255ar

DOI : https://doi.org/10.7202/027255ar

Aller au sommaire du numéro

\section{Éditeur(s)}

Société de philosophie du Québec

\section{ISSN}

0316-2923 (imprimé)

1492-1391 (numérique)

Découvrir la revue

Citer cet article

Nadeau, R. (1994). La philosophie des sciences après Kuhn. Philosophiques, 21(1), 159-189. https://doi.org/10.7202/027255ar

\section{Résumé de l'article}

En 1962, Thomas Kuhn fait paraître l'ouvrage qui allait le rendre célèbre, à savoir La Structure des révolutions scientifiques. Il visait à produire en philosophie des sciences ce quil appela une "gestalt switch ". Il entendait, en effet, mettre en cause le " paradigme épistémologique cartésien » et proposer que l'analyse logico-méthodologique cède définitivement la place à une approche historique et psychologique des sciences . Mon propos est de faire voir que, bien que les premiers critiques de Kuhn se soient le plus souvent fourvoyés, en proposant ce virage radical, Kuhn a amplifié sinon induit une crise profonde dans la philosophie des sciences post-positiviste. Car, ni l'histoire, ni la sociologie des sciences ne sont adéquatement outillées pour répondre aux questions spécifiquement logico-méthodologiques que la connaissance scientifique suscite. 


\title{
LA PHIILOSOPHIE DES SCIENCES APRÈS KUHN
}

\author{
par \\ Robert Nadeau
}

RÉSUMÉ : En 1962, Thomas Kuhn fait paraitre l'ouvrage qui allait le rendre célèbre, à savoir La Structure des révolutions scientifiques. Il visait à produire en philosophie des sciences ce qu'il appela une "gestalt switch . Il entendait, en effet, mettre en cause le "paradigme épistémologique cartésien " et proposer que l'analyse logico-méthodologique cède définitivement la place à une approche historique et psychologique des sciences. Mon propos est de faire voir que, bien que les premiers critiques de Kuhn se soient le plus souvent fourvoyés, en proposant ce virage radical, Kuhn a amplifié sinon induit une crise profonde dans la philosophie des sciences postpositiviste. Car, ni l'histoire, ni la sociologie des sciences ne sont adéquatement outillées pour répondre aux questions spécifiquement logico-méthodologiques que la connaissance scientifique suscite.

ABSTRACT: In publishing his 1962 book on The Structure of Scientific Revolutions, Thomas Kuhn became world famous. He wanted to provoke what he called a "gestalt switch" in philosophy of science. After having characterized the "Cartesian Paradigm" as the most prominent failure of our epistemology, Kuhn finally proposes that we switch from logic and methodology to history and sociology of science. I would like to show that, even if we grant that Kuhn's first critics were out of focus, in taking the Kuhnian turn, philosophy of science and epistemology runs into crisis. For, using exclusively historiographical and sociological concepts and types of investigation, epistemological and methodological analysis are no longer adequatly feasible. Transformed into historians and "metahistorians " of science, philosophers of the new Kuhnian persuasion are no longer apt to give proper treatment to those still legitimate concerns related to the strictly logical and methodological dimensions of scientific knowledge. 
L'heure des bilans a sonné en philosophie des sciences. On soutient généralement que, jusqu'aux années soixante, le néo-positivisme a largement dominé la scène de la philosophie des sciences ou de la méthodologie, et l'empirisme logique celle de l'épistémologie, mais qu'à partir de cette période, des revirements majeurs se sont produits. Une nouvelle philosophie des sciences, marquee entre autres choses par une plus grande ouverture à l'histoire et à la sociologie des sciences, aurait vu le jour, et une nouvelle épistémologie, anti-cartésienne ou anti-rationaliste, aurait commencé de prédominer. On a coutume de rattacher ces virages, voire ces ruptures, aux noms de Norwood Hanson ${ }^{\mathrm{I}}$, de Paul Feyerabend ${ }^{2}$, mais en particulier au nom de Thomas Kuhn. C'est de ce dernier dont il sera exclusivement question dans cet article. Et une seule préoccupation orientera mon propos : Kuhn a-t-il exercé une influence positive sur le développement de la philosophie des sciences contemporaine? L'examen de l'impact effectif et de la portée critique réelle des thèses contenues dans son ouvrage séminal de ig62, thèses reçues par plusieurs comme radicalement inacceptables parce qu'indéfendables ${ }^{3}$, exigerait sans doute l'espace ample d'une monographie plutôt que celui forcement restreint d'un article. Aussi n'est-ce pas à cet examen que je vais me livrer ici. Je me contenterai de soulever la question suivante : en supposant qu'on soit fondé de considérer qu'au

I. Hanson a publié en $195^{8}$ un ouvrage qui a été reçu comme marquant, à l'époque mais aussi subséquemment : Patterns of Discovery. An Inquiry into the Conceptual Foundations of Science. Cambridge, Cambridge University Press, mais il semble être depuis tombé dans l'oubli.

2. Le nom de Feyerabend, récemment décédé, a fait couler beaucoup d'encre à la suite de la publication de Against Method en 1975 (traduction française : Contre la méthode. Esquisse d'une théorie anarchiste de la connaissance, Paris. Seuil, 1979). Mais son œuvre ultérieure, notamment Science in a Free Society (Londres, 1978) n'a pas eu l'heur d'avoir autant de succès. Ses Philosophical Papers, qui ont été recueillis en 1981 ( 2 vols., Cambridge, Cambridge University Press), présentent pourtant encore beaucoup d'intérêt.

3. Les personnes intéressées à faire le point sur la philosophie des sciences de Kuhn, et en particulier sur la valeur des critiques qu'on a adressées à Kuhn, peuvent consulter deux ouvrages récents de grande qualité. D'abord, Reconstructing Scientific Revolutions. Thomas S. Kuhn's Philosophy of Science, de Paul Hoyningen-Huene (Chicago et Londres, The University of Chicago Press, 1993), traduction de l'édition allemande originale parue en 1989 . L'auteur de ce livre, docteur en physique théorique. enseigne la philosophie à l'Université de Constance, et pour préparer son ouvrage. Paul Hoyningen-Huene a passé un an au MIT auprès de Kuhn en 1984-85. On lira également l'ouvrage collectif World Changes : Thomas Kuhn and the Nature of Science édité par Paul Horwich (Cambridge, MIT Press, 1993), un recueil d'essais auxquels Kuhn fournit ensuite la réplique. 
tournant des années soixante, la philosophie des sciences d'obédience positiviste ou logico-empiriste ait été plus ou moins en crise, doit-on convenir que les prises de position de Kuhn ont contribué à résorber cette crise ou ne devrait-on pas plutôt avancer la thèse qu'elles ont davantage contribué à l'exacerber? En d'autres termes, en supposant que nous bénéficiions maintenant de suffisamment de distance critique pour pouvoir soulever la question et y voir clair, quelle postérité sied-il de reconnaître à Kuhn?

La philosophie contemporaine nous a légué jusqu'ici au moins deux sens fondamentaux du concept de «crise » appliqué à des questions d'ordre épistémologique et logico-méthodologique. L'un nous vient de Husser $l^{4}$ et met en cause l'absence d'entreprise fondationnelle adéquate, dont la conséquence logique à terme est le scepticisme. L'autre nous vient précisément de $\mathrm{Kuhn}^{5}$ et sert à caractériser certains épisodes du développement historique des sciences physiques au cours desquels, à la faveur d'une sorte de « conversion », une communauté scientifique donnée est amenée à rejeter son ancienne conception des choses et à mettre en place une nouvelle matrice disciplinaire. Le rapprochement entre ces deux acceptions du concept de crise de la connaissance n'est pas gratuit puisque, pour Kuhn également, les moments de crise sont les rares moments où les fondements des sciences en vigueur se trouvent mis en question par les praticiens d'une discipline donnée. C'est la notion kuhnienne de « crise » que j'aimerais d'abord dégager ici, d'une part parce qu'elle comporte un aspect essentiel que l'autre notion ne me paraît pas posséder, et d'autre part parce que le

4. Cf. Die Krisis der europäischen Wissenschaften und die transzendentale Phänomenologie. Trad. franç. de Gérard Granel : La crise des sciences européennes et la phénoménologie transcendantale, Paris, Gallimard, 1976).

5. Cf. The Structure of Scientific Revolutions, Chicago et Londres : The University of Chicago Press, $\mathrm{I}^{\text {ere }}$ éd., Ig62. La deuxième édition revue et augmentée d'une postface a paru en 1970 . Cet ouvrage a d'abord connu une première traduction française anonyme (La structure des révolutions scientifiques. Paris. Flammarion, Nouvelle bibliothèque scientifique, 1972), puis une seconde traduction par Laure Meyer, officiellement revue par Kuhn (Paris, Flammarion, Coll. « Champs », 1983). Cette seconde traduction a été grandement améliorée grâce, entre autres choses, à la minutieuse analyse de claude Savary qui avait suggéré de très nombreuses corrections à la première traduction (voir les remerciements de l'èditeur en p. 6). Le travail de Savary a été publie sous le titre « The Structure of Scientific Revolutions. Etude critique de la traduction française de Thomas S. Kuhn » dans la collection Recherches et Théories (Cahiers de recherche publiés par les départements de philosophie de l'UQAM et de l'UQTR, $\Pi^{\circ} 25,1982,74$ p.). Dans la suite de mon texte, je référerai à l'ouvrage de Kuhn par l'abréviation SRS que je citerai, à moins d'indication contraire, dans le texte de la traduction française de 1983 . 
programme de recherche de Kuhn lui-même me paraît avoir contribué à amplifier la crise de la philosophie des sciences post-positiviste. C'est, du moins, la thèse en faveur de laquelle j'entends argumenter.

Quel est donc ce trait fondamental propre à la notion kuhnienne de crise ? Il y a crise, selon Kuhn, quand le programme de recherche établi se révèle incapable de continuer à jouer son rôle, à savoir permettre de résoudre des problèmes spécifiques, ou encore quand les chercheurs eux-mêmes (car la base du système kuhnien, c'est la structure de groupe des communautés scientifiques) acquièrent la conviction que partie ou tout de l'ensemble des élements composant leur matrice disciplinaire qqui inclut les généralisations symboliques, les modèles métaphysiques et heuristiques, les valeurs et surtout les solutions exemplaires à certains problèmes clés) ne convient plus pour la poursuite de leurs travaux de recherche. Dans ce dernier cas, ce peut être, par exemple, soit parce que les problèmes y sont progressivement vus comme mal posés, soit encore parce que les normes standard de solution acceptées jusquelà sont atteintes de vices rédhibitoires. De telles situations permettent que soit rompu l'espèce de contrat social qui liait jusque-là les membres du groupe scientifique. Donc, si l'on se fie à la doctrine de Kuhn, il y a crise quand le changement de paradigme s'annonce pratiquement comme incontournable. Il y a crise quand, face à des difficultés apparaissant comme insurmontables si l'on en reste au statu quo, la possibilité, voire la nécessité de changer de tradition de recherche semblent acquises à suffisamment de chercheurs susceptibles d'en persuader d'autres qui, progressivement, formeront une nouvelle majorité dominante.

C'est précisément en ce sens que Kuhn se situe au cœur d'une crise dans la philosophie des sciences post-positiviste. Du reste, non seulement en est-il conscient, mais il en fait une revendication claire et explicite. Kuhn nous dit, en effet, que le « paradigme épistémologique traditionnel » (aussi qualifié par Kuhn de «paradigme cartésien ») a échoué à la tâche, et donc qu'il doit être remplacé par un autre encore à inventer ${ }^{6}$. Kuhn se propose en quelque sorte d'être l'instigateur de la solution de remplacement. Mon propos ici est de faire voir que, loin d'aboutir à la résorption d'une crise de la philosophie des sciences qu'il n'a pas lui-même engendrée, Kuhn a contribué à amplifier cette crise par certains aspects de son approche. La crise dont je parle ici est celle qui s'est installée en philosophie des sciences par la remise en question, au début des années soixante, de la « conception standard des théories

6. SRS, p. I70: Kuhn y parle du « paradigme formulé par Descartes qui s’est développé 
scientifiques », époque à partir de laquelle on fut porté à croire que la vision rationaliste traditionnelle de ce qu'est la science devait être révisée de fond en comble. Pour dire les choses en termes kuhniens, la prise en consideration de la structure dynamique de l'histoire des sciences révélerait l'existence de révolutions scientifiques : or de tels changements radicaux ne pourraient être expliqués ni à l'intérieur du paradigme épistémologique né avec la science moderne, ni à l'intérieur de la philosophie des sciences traditionnelle. La conclusion qui semble s'imposer à Kuhn est qu'il importe de révolutionner aussi bien l'épistémologie que la philosophie des sciences. Or, parce que cette révolution débouche sur une nouvelle forme d'historicisme, de psychologisme et de sociologisme, elle me paraît amplifier la crise qu'elle voudrait résoudre. Jaimerais faire voir que les analyses kuhniennes, s'il est vrai qu'elles ont renouvelé nos problèmes en philosophie des sciences, sont cependant inaptes à en fournir une solution acceptable.

\section{Le tournant des années soixante}

Mais demandons-nous d'abord ce qu'enseignait cette conception standard de la science. A défaut de pouvoir en fournir ici une analyse détaillée, j'en esquisserai seulement une figure globale. Dans la perspective standard, la science est approchée comme une entreprise intellectuelle systématique, contrôlée aussi bien par les vertus des algorithmes logiques que par l'appel à l'observation des faits. Le but de cette entreprise est d'arriver à formuler des assertions vraies à propos du monde conçu comme Nature, c'est-à-dire comme un système hièrarchique de régularités interdépendantes. Le type de vérité recherchée est tel que ces assertions prennent la forme de lois naturelles générales exprimant les régularités régissant la réalité, et cela de manière universelle et omnitemporelle. L'observation, et l'expérimentation lorsque la chose est possible, sont conçues comme les procédures qui donnent un accès privilégie aux faits empiriques que les hypothèses théoriques essaient d'expliquer, voire qu'elles permettent éventuellement d'anticiper. Ces

en même temps que la dynamique newtonienne et s'est révélée fort utile, tant dans le domaine de la science que de la philosophie l...l De nos jours, les recherches poursuivies dans certains domaines de la philosophie, de la psychologie, de la linguistique et même de l'histoire de l'art tendent à suggérer que quelque chose ne va pas dans le paradigme traditionnel. » Kuhn poursuit de la manière suivante: «Aucun de ces problèmes créateurs d'un état de crise n'a encore produit une théorie viable, susceptible de remplacer le paradigme épistémologique traditionnel, mais ils commencent à nous suggérer ce que seront certains des caractères de ce paradigme $\gg$ (ibid). 
hypothèses théoriques, formulant des lois générales, sont conçues comme pouvant être hierarchiquement ordonnées en fonction de leur plus ou moins grande portée. Au bas de la hiérarchie se trouvent les généralisations empiriques (appelées aussi « lois expérimentales » ou encore « lois phénoménologiques »), et au sommet les « lois théoriques $\gg$ les plus fondamentales, c'est-à-dire celles qui permettent la dérivation des lois théoriques secondaires. Ainsi, les lois expérimentales sont exprimées dans un langage observationnel faisant immédiatement référence aux objets et processus tels qu'ils sont perçus, alors que les lois théoriques, pour leur part, sont exprimées dans un langage plus abstrait, le plus souvent mathématisé, et postulant l'existence d'entités, d'éléments ou de fonctions à proprement parler inobservables.

De la sorte, les lois théoriques, au contraire des généralisations issues de l'observation ou de l'expérimentation, ne seraient pas susceptibles d'une preuve empirique directe. Du reste, la fonction de ces lois théoriques n'est pas d'exprimer sous forme de généralités les phénomènes observés, mais plutôt d'expliquer les lois mêmes qui régissent de telles régularités. Dans cette perspective, une loi expérimentale est considérée comme expliquée pour autant qu'elle soit logiquement dérivable d'une ou de plusieurs lois théoriques par ailleurs bien confirmées, tout comme un théorème de géométrie est dérivable d'un ensemble de postulats, d'axiomes ou de théorèmes déjà prouvés, les termes utilisés étant tous définis, sauf certains termes primitifs, et les règles d'inférence valide étant convenues au préalable. Les lois théoriques ne sont donc, dans cette perspective, qu'indirectement testables par observation et expérimentation : et pour autant qu'il soit considéré que l'une au moins des conséquences observables d'une telle loi ne correspond pas au résultat anticipé, cette loi théorique peut être tenue pour infirmée, à moins que l'on ne soit en mesure de l'ajuster par le recours à un ensemble d'hypothèses auxiliaires acceptables, c'est-à-dire testables indépendamment de l'hypothèse théorique principale. Mais la fonction première de ces lois théoriques est d'unifier graduellement les divers champs du savoir. Il est admis comme possible en principe d'inclure par un processus de réduction une certaine théorie dans une autre plus génerale dont elle serait déductible, ce qui suppose évidemment que toutes ces théorisations soient opérées dans un langage commun ou qu'elles soient faites du moins dans des langages intertraduisibles.

C'est pourquoi, dans la perspective qui a dominé la philosophie des sciences jusqu'au début des années soixante, on suppose que lorsqu'une théorie scientifique est historiquement supplantée par une autre, les faits expliqués par elle jusque-là, loin d'être passés sous silence, sont dorénavant expliqués par la nouvelle théorie. La nouvelle 
théorie se trouve donc à englober la première au sens où elle rend compte des faits dont la première rendait compte. Qui plus est, cette nouvelle théorie permet également d'expliquer des faits qui ne sont pas ou ne pouvaient pas être expliqués par l'ancienne théorie. Ce mécanisme de progrès par corrections successives et par inclusion englo bante rend compte de l'effet de cumul du savoir que l'histoire des sciences permet d'observer. Ainsi donc, alors que, sur le plan du langage théorique, les sciences sont vues comme se transformant de manière relativement discontinue, c'est-à-dire par bonds en avant et par sauts qualitatifs, sur le plan du langage observationnel, elles sont vues comme continues, c'est-à-dire comme additionnant leurs explications les unes aux autres, permettant ainsi que de plus en plus de phénomènes soient « sauvés ». Par conséquent, si l'on s'en remet à la conception standard des theories de la science empirique, à travers le flux des croyances scientifiques mouvantes, il y aurait malgré tout croissance de la connaissance et donc progrès constant de notre connaissance du monde environnant, et en particulier du monde naturel. De plus, à travers les manifestations théoriques diverses révélées dans l'histoire des sciences se profileraient une même logique et une unique méthode qu'il appartiendrait à l'épistémologue et au méthodologue de mettre au jour. Voilà, en bref, la « conception orthodoxe » (Herbert Feigl), la « received view » (Frederick Suppe) ou la «standard view » (Israel Scheffler), et ce que, de façon plus globale, j'ai choisi d'appeler ailleurs « le Modèle Épistémologique Standard ${ }^{7}$. Car il en va ici non seulement d'une conception de la science mais aussi et surtout d'une assignation de tâche à l'épistémologie et à la philosophie des sciences conçue comme analyse logique et méthodologique.

Cela dit, comment convient-il de caractériser maintenant l'apport fondamental de Kuhn à la philosophie des sciences à partir de la période qui s'ouvre au tournant des années soixante? À mon avis, Kuhn n'a pas seulement provoqué une formidable révision du concept de science, il a également redéfini la table des matières du manuel de base en philosophie des sciences, si l'on peut s'exprimer ainsi. Mais il m'apparaît essentiel de distinguer ces deux aspects de la question. La thèse centrale du présent article est que ce n'est pas d'abord parce qu'il a contesté la vision établie de la science que Kuhn a contribué à amplifier la crise de la philosophie des sciences contemporaine, mais parce qu'il a œuvré à

7. Cf. « Problématique de la preuve en épistémologie contemporaine », Philosophiques, VII, 2 (octobre 1980), p. 217-246. 
substituer à cette entreprise disciplinaire telle qu'elle a été menée au cours des cent dernières années un tout autre programme de recherche qui conteste la valeur et l'utilité, voire même la légitimité de l'analyse épistémologique et logico-méthodologique des sciences. Car, l'examen des tenants et aboutissants du programme de recherche kuhnien montrera, du moins c'est ce que je souhaite, que cette approche, pour révolutionnaire qu'elle soit, vise à toutes fins utiles à remplacer l'analyse logico-méthodologique des théories scientifiques par l'analyse sociohistorique des pratiques scientifiques. Il n'y a donc pas que l'image de la science qui se trouve bouleversee par Kuhn : la philosophie des sciences traditionnelle elle-même se trouve au bout du compte déboutée de toutes ses prétentions. Toute la question est de savoir si les arguments qu'on tire de Kuhn pour procéder à cet aggiomamento sont justifiés. Et avant de faire le point sur les critiques qu'on a opposées à la doctrine de Kuhn, essayons de mieux comprendre en quoi cette doctrine est en rupture avec la philosophie des sciences qui prédominait encore au tournant des années soixante.

Certes, il est facile de soutenir aujourd'hui que les canons du positivisme logique ont fait long feu : beaucoup de tapage pour rien. diraient certains en signe de dérision. Car, si le pilier central de cette philosophie devait être constitué par la doctrine de la signification cognitive des termes théoriques, force est de constater, comme l'affirme lui-même l'un des principaux protagonistes de cet épisode de l'histoire de la philosophie des sciences contemporaine, à savoir Carl Hempel, que l'entreprise a lamentablement échoué ${ }^{8}$. L'insuccès de cette philosophie des sciences en matière de sémantique n'est certainement pas pour rien dans ses avatars ultérieurs, d'autant qu'il faut reconnaitre que l'un des principaux fronts sur lesquels Kuhn a précisément tenté d'effectuer une percee est celui de la sémantique des théories physiques, avec, au cœur de la problématique redessinée par lui, l'épineuse question de l'incommensurabilité des paradigmes ou, comme il préférera le dire par la suite, des « matrices disciplinaires ».

Mais, à moins de penser que l'empirisme logico-positiviste a tout bonnement disparu de la scène pour cause d'inadaptation à l'environnement scientifique, la question se pose de savoir qui, au juste, a mis

8. Cf. Carl G. Hempel, « Empiricist Criteria of Cognitive Significance : Problems and Changes » (dans Aspects of Scientific Explanation, New York, Macmillan, 1965, p. 101122), traduit en français par Pierre Jacob, « Les critères empiristes de la signification cognitive : problèmes et changements » (dans De Vienne à Cambridge. L'héritage du positivisme logique de 1950 à nos jours, Paris, Gallimard, 1980, p. 6r-86). 
fin à ses jours. Karl Popper l'aurait-il assassiné, ainsi qu'il l'a prétendu lui-même ${ }^{9}$, ou serait-ce plutôt Thomas Kuhn, comme d'autres l'ont affirmé $^{\text {IO }}$ ? On semble avoir oublié que l'ouvrage de Kuhn, dont on a dit qu'il consommait définitivement la rupture avec le positivisme logique, a vu le jour dans le cadre de la collection de l'Encyclopedia of Unified Science dirigée par Otto Neurath, Rudolf Carnap, et Charles Morris ${ }^{\mathrm{II}}$. On peut prétendre sans grand risque de se tromper que cet ouvrage n'avait pas été planifié par son auteur comme l'ouvrage qui devait donner un formidable coup de boutoir à l'empirisme logique ${ }^{12}$. Il est clair que Kuhn lui-même pense avoir développé une argumentation très critique de la doctrine logico-empiriste ${ }^{13}$. Mais comment Kuhn se représente-til les lignes de force suivies dans le développement de sa philosophie des sciences après 1962 ? Signant récemment l'avant-propos d'un

9. Voir la section XVII de son autobiographie intellectuelle intitulée « Qui a tué le positivisme logique? » (Ia Quête inachevée, Paris, Calmann-Lévy, 198I, P. I25-128).

10. L'acte d'accusation fait l'objet d'un minutieux examen de la part de George A. Reisch dans « Did Kuhn Kill Logical Empiricism? » (Philosophy of Science 58, I99ז, p. 264-277).

II. L'ouvrage The Structure of Scientific Revolutions a effectivement vu le jour en 1962 dans la « International Encyclopedia of Unified Science »: cf. Foundations of the Unity of Science, vol. II, n' 2, Chicago et Londres : The University of Chicago Press. 1962.

12. On lira à ce propos la formidable analyse de Frederick Suppe « The Search for Philosophic Understanding of Scientific Theories » dans The Structure of Scientific Theories, F. Suppe, ed. (Urbana : University of Illinois Press, 1974, reed. 1977. P. I241).

13. L'œuvre publiée de Thomas Kuhn s'étend de 1945 à 1993. Ses livres sont: The Copernican Revolution : Planetary Astronomy in the Development of Western Thought (Cambridge : Harvard University Press; trad. franç- par Avram Hayli : La Révolution copemicienne, Paris. Fayard, Collection « Le phénomène scientifique », 1973); The Structure of Scientific Revolutions, op. cit; The Essential Tension, Selected Studies in Scientific Tradition and Change (Chicago et Londres : The University of Chicago Press. 1977; cet ouvrage comprend douze articles précédemment publiés et deux inédits. Traduction française par Michel Biezunski, Pierre Jacob, Andrée Lyotard-May et Gilbert Voyat: La Tension essentielle. Tradition et changement dans les sciences, Paris, Gallimard, Bibliothèque des sciences humaines, 1990); et Black Body Theory and the Quantum Discontinuity, I894-Ig12 (Oxford : Clarendon, 1978). Outre les articles recueillis en 1977 dans La Tension essentielle, Kuhn a fait paraître les principaux articles suivants : "The Halt and the Blind : Philosophy and History of Science » (British Joumal for the Philosophy of Science, 31, 1980, p.18I-192); « What are Scientific Revolutions ? » (dans L. Krüger, L. J. Daston et M. Heidelberger, eds., The Probabilistic Revolution. Vol. I, Ideas in History. Cambridge. MIT Press, 1987, p. 7-22); "Commensurability, Comparability, Communicability (dans PSA 1982. Proceedings of the 1982 Biennial Meeting of the Philosophy of Science Association, P. D. Asquith et T. Nickles, eds., East Lansing, Philosophy of Science Association, 1983. 
ouvrage $^{14}$ en tous points remarquable sur son cuvre élaborée sur plus de trente ans, Kuhn fait remarquer qu'après la publication en 1962 de l'ouvrage qui allait le rendre célèbre ${ }^{\mathrm{I5}}$, il mit l'accent sur trois points particuliers de sa doctrine, entraînant de la sorte un déplacement du centre d'intérêt des philosophes des sciences et des épistémologues. Pour les besoins de cet exposé, je les mentionnerai dans l'ordre inverse de celui que suit Kuhn. Premièrement, avouant avoir abusé dans SRS de l'idée qu'une révolution scientifique constituait une sorte de « gestalt switch », c'est-à-dire un changement brusque et radicalement discontinu dans la perception de la réalité. Kuhn reconnaît avoir prêté de la sorte aux groupes scientifiques, à savoir les véritables véhicules des révolutions scientifiques, une opération qui ne peut être le fait que des seuls individus chercheurs ${ }^{16}$. Selon Kuhn, une partie importante de ses travaux a par la suite consisté à corriger cette confusion entre ce qui est d'ordre individuel et ce qui est d'ordre collectif, de manière à mieux faire ressortir qu'une révolution scientifique est le fait des membres d'une communauté scientifique agissant sur une base individuelle, et

p. 669-688); « Rationality and Theory Choice » Uoumal of Philosophy, 80, 1983. P. 563570); « Possible Worlds in History of Science » (dans S. Allén, ed., Possible Worlds in Humanities, Arts, and Sciences, Berlin, de Gruyter, 1989, p. 9-32); «Dubbing and Redubbing : the Vulnerability of Rigid Designation 》 (dans C. W. Savage, ed., Scientific Theories, Minnesota Studies in Philosophy of Science 14, Minneapolis : University of Minnesota Press, 1990, p. 298-318); « The Road since Structure » (dans A. Fine, M. Forbes, et L. Wessels, eds., PS A Iggo, Proceedings of the Iggo Biennal Meeting of the Philosophy of Science Association, vol. 2, East Lansing : Philosophy of Science Association, 1991, p. 2-13); « The Natural and the Human Sciences 》(dans D. R. Hiley, J. E. Bohman, et R. Shusterman, eds., The Interpretive Turn, Ithaca, Cornell University Press, 199., p. 17-24); et finalement « Afterwords 》(dans Paul Horwich, ed., World Changes: Thomas Kuhn and the Nature of Science, Cambridge, MIT Press, ig93. P. 3ir-34r). Signalons également la publication sous forme de cahier de recherche de « The Trouble with the Historical Philosophy of Science », une confërence (la « Robert and Maurine Rothschild Distinguished Lecture ») prononcée le ig novembre iggr à la Harvard University (Cambridge, An Occasional Publication of the Department of the History of Science, 1992).

14. Cf. Paul Hoyningen-Huene, Reconstructing Scientific Revolutions, op.cit., p. x⿺-xaII.

15. De tous les ouvrages de philosophie des sciences, le livre de Kuhn est très certainement celui qui a été le plus vendu jusqu'ici. On dit aussi qu'il est en général, de tous les ouvrages de philosophie, celui qui se trouve le plus souvent cité. Le tirage de la seule édition en langue anglaise approche maintenant le million d'exemplaires, sans compter que cet ouvrage a été jusqu'ici traduit en dix-neuf langues différentes.

16. Kuhn a effectivement illustré ce qu'il entendait par « révolution scientifique » par l'expérience de la transformation de la perception où une certaine figure apparaissant d'abord comme un lapin devient soudainement un canard. 
donc éventuellement divergente, mais tout en insistant sur le fait qu'une révolution scientifique n'est véritablement advenue que lorsqu'une communauté organisée de chercheurs a dans son ensemble et pour l'essentiel changé radicalement de point de vue et de façon de faire. En deuxième lieu, c'est à la question de l'incommensurabilité et au problème de la communication partielle entre les scientifiques appartenant à des communautés de recherche distinctes que Kuhn dit s'être le plus attardé dans la période ouverte par la publication de SRS. Et il n'est pas faux de prétendre que cette problématique est aussi celle qui semble avoir reçu le plus d'attention dans la littérature des années soixante-dix et quatre-vingts. Il serait d'ailleurs présomptueux de prétendre qu'aujourd'hui cette question est réglée et donc qu'elle est derrière nous ${ }^{17}$. Enfin, et c'est ce point que Kuhn place en tête des préoccupations qui furent centrales pour lui après ig62, la question de savoir quelle contribution la SRS entendait faire précisément à la philosophie des sciences proprement dite devait recevoir une clarification. Sans nier que son ouvrage ouvrait la porte à des considérations de nature proprement historiographique et qu'il pavait sans doute la voie à un renouvellement de l'analyse sociologique des sciences, Kuhn insiste pour dire que ses efforts ultérieurs ont grandement consisté à retrouver l'inspiration proprement philosophique qui l'avait d'abord animé. Suivant Kuhn, cette inspiration est liée à l'idée que les groupes que forment les scientifiques pour exercer leur profession de chercheur sont fondamentalement des « communautés

17. Il serait long et fastidieux de répertorier tout ce qui s'est publié d'important sur cette question depuis dix ou quinze ans. On aura néanmoins une bonne idée des discussions en cours en consultant $X$. Chen, « Local Incommensurability and Communicability » (dans A. Fine, M. Forbes et L. Wessels, eds, PSA rggo. Proceedings of the 1990 Biennial Meeting of the Philosophy of Science Association, vol. I. East Lansing, Philosophy of Science Association, 1990, p. 67-76), J. Hintikka, « On the Incommensurability of Theories 》 (Philosophy of Science 55, 1988, p. 25-38). H.C. Hung, «Incommensurability and Inconsistency of Language » (Erkenntnis 27, 1987. p. 323-352), N.J. Nersessian, « Scientific Discovery and Commensurability of Meaning » (dans K. Gavroglu, Y. Goudaroulis et P. Nicolacopoulos. eds., Imre Lakatos and Theories of Scientific Change, Dordrecht, Kluwer, 1989. P. 323-334), D. Pearce, «The Problem of Incommensurability : A Critique of Two Instrumentalist Approaches » (dans I. Hronszky, M. Fehér et B. Dajka, eds., Scientific Knowledge Socialized. Selected Proceedings of the 5 th International Conference on the History and Philosophy of Science Organized by the IUHPS. Dordrecht, Kluwer, I988, p. 385-398) et finalement D. B. Wong, « Three Kinds of Incommensurability » (dans M. Krausz, ed., Relativism : Interpretation and Confirmation, Notre Dame, Notre Dame University Press, 1989, p. 140-158). 
de langage ou de discours $\gg^{18}$, et c'est dans le cadre de l'analyse de cette réalité que doivent être formulées et traitées les questions de l'incommensurabilité des théories, de la communication partielle entre savants venant d'horizons de recherche différents, et de l'« ontologie professionnelle » à laquelle, diversement, les uns et les autres adhèrent. Mais avant de se demander si la percée effectuée par Kuhn avec SRS a effectivement été vue avec le temps comme débouchant sur une nouvelle philosophie de la connaissance scientifique, demandonsnous comment, au départ, les philosophes qui furent les premiers critiques de Kuhn ont réagi à sa theoorie.

\section{Kuhn et ses premiers critiques : une lecture ratée}

Les commentateurs récents de Kuhn ont bien mis en lumière à quel point la doctrine présentée par Kuhn dans SRS a été le plus souvent travestie pour être plus facilement soumise à la critique. Cest pourquoi, avant d'ajouter au stock déjà passablement considérable de critiques adressées de toutes parts à Kuhn, j'aimerais d'abord me démarquer de ceux que la publication de la Structure des révolutions scientifiques semble avoir outragés. Car on a fait dire à Kuhn des choses qu'il n'a jamais dites, et prenant prétexte des formulations ambiguës, des notions équivoques lau premier chef desquelles il faut compter le concept de " paradigme » lui-mème ${ }^{\mathrm{I9}}$ ), ou encore des thèses plus ou moins obscures $^{20}$ qu'on peut trouver dans l'ouvrage publié par Kuhn en 1962, on s'est acharnè à faire de lui un « irrationaliste subjectiviste relativiste ». Considérons tour à tour ces trois critiques de Kuhn et faisons-le distinctement comme si elles ne formaient pas dans le fond la trame d'une seule et même critique.

La charge d'irrationalisme est gratuite si l'on considère que Kuhn n'a jamais soutenu que le choix entre théories rivales équivalait à une

I8. Cf. Foreword, loc cit., p. xur.

19. Il faut consulter à ce propos la magistrale ettude de Margaret Mastermann, « The Nature of a Paradigm » (dans Criticism and the Growth of Knowledge. Imre Lakatos et Alan Musgrave, eds. (Londres : Cambridge University Press, 1970, P 59-89).

20. Je me contenterai de ne donner qu'un exemple de formulation qui prête flanc à la critique ou qui ouvre la porte à la contestation : « Jai, par exemple, écrit Kuhn, une conscience aiguë des difficultés créêes si l'on dit que lorsque Aristote et Galilée regardaient des pierres qui se balançaient, le premier voyait une chute entravée et le second un pendule. Les mêmes difficultés, sous une forme encore plus fondamentale, sont soulevées par les premières phrases de ce chapitre : bien que le monde ne change pas après un changement de paradigme, l'homme de science travaille dans un monde différent » (SRS, p. I7o). 
décision qui ne devait rien à l'échange d'arguments sensés ou rationnels entre partisans de théories mutuellement incompatibles. Kuhn insiste seulement pour dire que la persuasion, peu importe, du reste, laquelle des théories concurrentes finira par l'emporter, ne saurait s'expliquer totalement et exclusivement en termes de dialogue rationnel ou d'échanges d'arguments dont seul le contenu logique importerait, comme le voudraient par exemple Karl Popper et ceux que Gerard Radnitzky et Gunnar Andersson ont identifiés comme etant les partisans de la LSE Position ${ }^{21}$. L'adhésion à une matrice disciplinaire n'est donc pas, aux yeux de Kuhn, une pure affaire de raison logique car elle est aussi une affaire de « conversion du regard ». En utilisant la notion de « conversion », Kuhn lançait un défi au rationalisme de type cartésien dans la mesure où, suivant sa doctrine, le processus de la transformation socio-historique du savoir scientifique est basé non pas tant sur l'argumentation que sur une sorte de transmutation des valeurs qui s'opère de temps à autre chez les scientifiques, dans des moments particulièrement cruciaux du développement des disciplines de pointe ou, pour utiliser l'expression de Kuhn, des « sciences matures 》.

La charge de subjectivisme est aussi trompeuse, puisque Kuhn prétend n'avoir rien dit qui laisserait penser qu'il n'existe pas de critères d'évaluation objectifs permettant de guider les scientifiques dans leurs décisions théoriques. Kuhn reconnait, en effet, qu'il existe au moins cinq critères jouant ce rôle, et il en parle comme de critères omniprésents, voire de critères constitutifs de l'activité scientifique en tant que telle $^{22}$. Ce que Kuhn soutient par contre, c'est que ces critères, loin de constituer des régles - comme si un algorithme de la justification du choix entre theories concurrentes était possible - doivent plutôt être considérés comme des valeurs susceptibles de définitions et d'applications diverses. Qui plus est, ces valeurs peuvent entrer en conflit les unes avec les autres et forcer les chercheurs à les hiérarchiser, voire à en abandonner une aux dépens d'une autre - par exemple, sacrifier la simplicité à la précision ou la portée à la cohérence. C'est donc le chercheur individuel qui, en dernière instance, en fonction de sa

2I. Il s'agit de John Watkins, John Worrall, Elie Zahar et Peter Urbach. Cf. G. Radnitzky et G. Andersson, eds., Progress and Rationality in Science (Dordrecht, Reidel, 1978).

22. La liste des critères d'évaluation comprend au moins, selon Kuhn, les cinq suivants : la précision [accuracy], la cohérence ou l'absence de contradiction interne Iconsistancel, l'envergure ou la portée [scopel, la simplicité et la fécondité lfruitfulness]. Cf. « Objectivité, jugement de valeur et choix d'une théorie » (dans La tension essentielle, op. cit.. chapitre XIII, p. 424-449). p. 426 et sq.; $c$. aussi la « Postface - 1969 ». dans SRS, p. 251²54. 
personnalité propre et nonobstant le fait qu'il soit partie intégrante d'un groupe, modulera et appliquera diversement ces critères-valeurs. Cela revient à dire que, bien que ces critères-valeurs soient à peu près les mèmes pour tous, il est tout à fait plausible et rationnel du point de vue de la survie du groupe de recherche qui sous-tend le développement d'une discipline que tous les scientifiques n'effectuent pas exactement le même choix au même moment. La diversité des voies protège le groupe de chercheurs - ou la communauté scientifique dans son ensemble, pourrait-on dire - contre les culs-de-sac possibles, contre les erreurs fatales et donc contre l'extinction, tout comme elle permet également à ceux qui font les meilleurs choix d'obtenir plus de reconnaissance et d'avoir une postérité que les autres n'auront pas.

Quant à la charge de relativisme, elle est liée à deux thèses fondamentales développées par Kuhn et qui méritent assurément qu'on les examine une à une. Ia première thèse défendue par Kuhn en cette matière est celle de la « theory-ladenness of observation " : c'est l'idée que toute observation est déterminée par la théorie même qu'elle entend servir à confirmer. Cette thèse de Kuhn, qui ne lui est d'ailleurs pas propre ${ }^{23}$, signifie à toutes fins pratiques qu'il n'existe pas de langage observationnel neutre dans lequel pourraient être formulées toutes les conséquences observables de théories incompatibles entre elles. Contrairement à ce que le modèle épistémologique standard a présupposé, il n'existe aucune possibilité de reformuler toutes les observations scientifiques dans un seul et même langage non théorique d'observation et de comparer sur cette base toutes les théories disponibles (et encore moins toutes les théories possibles, qui sont en nombre infini) pour pouvoir les départager selon leur mérite respectif. Car, suivant Kuhn, il est à toutes fins utiles impossible de distinguer rigoureusement, comme Carnap proposait de le faire ${ }^{24}$, entre langage d'observation et langage theorique : toute observation se formule en supposant d'abord acquise une certaine perspective théorique, sans laquelle les

23. C'est d'abord Hanson (op. cit.) qui a clairement mis ce point en évidence. Mais on trouve une thèse fort ressemblante chez Popper également. Seulement, suivant la distinction faite par Wolfgang Stegmüller entre deux formes de la thèse de la détermination de l'observation par la théorie, Popper opterait pour la plus faible et Kuhn pour la plus forte. $C f$. « Accidental ("non-substantial") Theory Change and Theory Dislodgment », dans Butts and Hintikka, eds., Historical and Philosophical Dimensions of Logic, Methodology, and Philosophy of Science, Dordrecht, Reidel (1977). p. 269-288. La distinction invoquée ici se trouve faite à la p. 277 (section 5).

24. Cf. « The Methodological Character of Theoretical Concepts », Minnesota Studies in the Philosophy of Science I, 1956, P. $3^{8-76 .}$ 
questions qu'on se pose en sciences et les problèmes qu'on tente de résoudre ne se poseraient pas ou du moins ne se présenteraient pas de la même manière. Un certain cadre théorique doit avant tout être construit au moins par hypothèse, une hypothèse que le chercheur doit supposer suffisamment plausible, légitime, et correcte. La thèse de Kuhn est que ce cadre non seulement decoupe l'observable mais que, en fournissant un système de catégories, il fait en quelque sorte émerger l'observable de nulle part et le rend exprimable. Vu ainsi, le cadre théorique exerce une contrainte permettant de fixer ce qui compte comme faits d'observation. Ce n'est donc pas l'observation seule, contrairement à ce qu'a enseigné traditionnellement l'épistémologie de la connaissance scientifique, mais bien l'observation et l'expérimentation situées dans un cadre conceptuel particulier et déterminant, qui permettent aux chercheurs d'opter progressivement pour la théorie qui constitue à leurs yeux non pas la meilleure approximation de la vérité, mais la meilleure garantie de succès pour résoudre les problèmes scientifiques déjà identifiés par eux et aussi ceux qui ne manqueront pas de se poser dans le développement futur de leurs travaux de recherche. Que cette option en faveur d'une perspective théorique n'intervienne pas comme interviendrait une pure décision rationnelle qui serait prise exclusivement sur la base d'un stock d'observations théoriquement neutres, cela n'implique pas qu'elle soit pour autant complètement arbitraire. Mais cette option de la communauté scientifique dans son ensemble en faveur d'un certain modèle théorique ne peut avoir lieu que si au départ certains chercheurs ont cru, sans avoir de preuves formelles et incontestables (Kuhn n'hésite pas à dire que c'est une question de « foi »). que cette avenue de recherche leur offrait le meilleur potentiel de rendement.

Quant à la seconde thèse fondamentale sur laquelle me paraît reposer ce que d'aucuns ont appelé le relativisme de Kuhn, c'est celle selon laquelle il ne saurait $\mathrm{y}$ avoir qu'une «communication partielle» entre scientifiques appartenant à des groupes disciplinaires distincts ou encore se situant à des époques différentes - ou suffisamment distantes l'une de l'autre - de l'histoire d'une discipline en particulier. Cette thèse est celle de l'« incommensurabilité des paradigmes », c'està-dire de l'incomparabilité au moins partielle des théories rivales, une thèse qui, assez malencontreusement pour Kuhn, a été assimilée à une thèse différente. à savoir la thèse anarchiste de Feyerabend. Cette dernière thèse de Kuhn me paraît mériter d'autant plus d'attention qu'elle va à contre-courant d'une idéologie «interdisciplinariste » un peu facile, mais toujours très à la mode. Si les univers de pensée des scientifiques sont relativement étanches d'une discipline à l'autre et d'une 
époque de la science à une autre, il n'y a pas lieu de croire qu'il existe un langage de la science qui soit universel ou que toutes les conceptions theoriques qui peuplent l'histoire des sciences soient comme les morceaux épars d'un seul et même puzzle ou encore comme les épisodes singuliers d'une hypergenèse de l'Esprit Scientifique. A moins de fournir une image du développement socio-historique des sciences qui soit différente de celle-ci, la charge de relativisme ne rime à rien. Elle ne fait que mettre une étiquette sur une doctrine dont il resterait à faire voir la fausseté ou l'incohérence, ce qui n'a jamais été fait que je sache.

Je n'endosse donc pas, pour ma part, les critiques formulées, et le plus souvent à l'emporte-pièce, contre le système de Kuhn, d'où qu'elles soient venues ${ }^{25}$. Car, dans la mesure où elles étaient le plus souvent le résultat de malentendus ou de ce que Kuhn n'hésite pas à appeler une « incompréhension radicale » de ses propos, et quelle que soit la part de responsabilité de Kuhn lui-même dans cette situation, les critiques

25. Ne pouvant répertorier ici toutes les critiques dont Kuhn a fait l'objet, je me contenterai de signaler certains des textes qui ont d'entrée de jeu donné le ton : S. Amsterdamski, Between Experience and Metaphysics (Dordrecht, D. Reidel, Synthese Library, vol. 77, Boston Studies in the Philosophy of Science, vol. xxxv, 1975, chap. v1: «Reformation and Counter-Reformation : Paradigms and Research Programs », p. 115-143); K.R. Popper, « Normal Science and its Dangers » (dans Criticism and the Growth of Knowledge, op.cit., p. 51-58); K.R. Popper, « Replies to my Critics » (dans The Philosophy of Karl Popper, P. A. Schilpp, ed., The Library of Living Philosophers, Vol. XIV, 2 vols, LaSalle Ill., Open Court, 1974, vol. II, Partie 3, section 39 : « Kuhn on the Normality of Normal Science », p. II44-1148, et section $40:$ : Wisdom on the Similarity between Kuhn and Popper », p. Ir48-1153; I. Scheffler, Science and Subjectivity (Indianapolis, New York, Kansas City, Bobbs-Merrill Co., I967 ( $2^{\mathrm{e}}$ édition 1978), chap. 4 : « Change and Objectivity », p. 67-89); I. Scheffler, « Vision and Revolution : A Postcript on Kuhn » (Philosophy of Science, 39, 1972, p. 366-374); D. Shapere « Review of The Structure of Scientific Revolutions, by T.S. Kuhn » (Philosophical Review, 73, 1964, p. 383-394); D. Shapere, « Meaning and Scientific Change » (dans Mind and Cosmos: Essays in Contemporary Science and Philosophy, R. Colodny, ed., University of Pittsburgh, Series in the Philosophy of Science, 1966, 3. p. 41-84); D. Shapere, The Legacy of Logical Positivism, Studies in the Philosophy of Science (Baltimore, The Johns Hopkins University Press, 1969, « The Approach of Feyerabend and Kuhn », p. 119-125); D. Shapere, « The Paradigm Concept » (Science, 172, 1971, p. 706-709); D. Shapere, « Notes Toward a Post-Positivistic Interpretation of Science, Part $\mathbf{I}$ » (dans Reason and the Search for Knowledge, Investigations in the Philosophy of Science. Dordrecht, Reidel, 1984, p. 102-110); S. Toulmin, « Discussion» sur "The Function of Dogma in Scientific Research", de T. S. Kuhn » (dans Scientific Change : Historical Studies in the Intellectual, Social and Technical Conditions for Scientific Discovery and Technical Invention, from Antiquity to the Present, A. C. Crombie, ed., Londres, Heinemann, 1963. P. 382-384); S. Toulmin, « Does the Distinction Between Normal and Revolutionary Science Hold Water? » (dans Criticism and the Growth of Knowledge, op. cit.. P. 39-47). 
en question visaient surtout à mal faire paraître les idées de Kuhn au nom d'une conception de la science que l'on voulait à tout prix tenir pour intangible. Et c'est presqu'en s'inventant un Kuhn fantôme, un homme de paille en quelque sorte, que les auteurs de ces différentes critiques plus ou moins acerbes ont tenté de parvenir à leurs fins. C'est pourquoi je n'hésite absolument pas, pour ma part, à déclarer que beaucoup de ces critiques sont de pures élucubrations et qu'elles procèdent d'une lecture de l'œuvre de Kuhn qui s'avère complètement ratée. Les premières critiques de Kuhn ont donc èté marquées au coin de l'incompréhension la plus flagrante, c'est le moins que l'on puisse dire. Mais si l'on peut reconnaître aujourd'hui que certaines interpretations de Kuhn équivalaient à un travestissement de sa pensée, cela ne veut pas dire qu'il eût été facile d'en rendre compte fidèlement. La chose est davantage perilleuse qu'il n'y paraît de prime abord. Et puisque je voudrais, pour ma part, ultimement déboucher sur une lecture plutôt critique de la contribution de Kuhn à la philosophie des sciences actuelle, je m'emploierai d'abord à montrer en quoi la philosophie de Kuhn ne peut pas être vue comme un rejet pur et simple de la rationalité de l'entreprise scientifique.

Il n'est pas complètement faux de dire qu'un certain «paradigme épistémologique traditionnel », que Kuhn caractérise comme étant «cartesien », a largement dominé la scène à peu près jusqu'à aujourd' hui. Reconnaissons avec Kuhn que c'est sous l'influence de cette conception de la connaissance que les doctrines méthodologiques traditionnelles, y compris celle allant de pair avec le positivisme logique, ont développé et maintenu un caractère individualiste et solipsiste très marque $^{26}$. Ce solipsisme est à ce point constitutif et fondamental de l'épistémologie traditionnelle que toute la question de la rationalité de la connaissance scientifique, notamment au cours des épisodes d'affrontement entre théories rivales, a toujours été posée comme une question relevant du comportement de l'individu chercheur ou de l'individu savant. C'est en quelque sorte en son âme et conscience, a-t-on souvent soutenu, que le scientifique doit se demander, en évaluant les mérites respectifs de théories concurrentes et incompatibles, laquelle est préférable du point de vue d'un certain nombre de critères de jugement objectifs incontournables. Du point de vue qui se trouve favorisé par une telle approche individualiste, il est clair, puisque la chose est implicite dans le fait que « le bon sens soit la chose du monde la mieux

26. Kuhn insiste d'ailleurs résolument sur cet individualisme solipsiste dans son avant-propos à l'ouvrage de Hoyningen-Huene (cf. loc. cit., p. xiII). 
partagée », que si, pour un individu rationnel donné, la rationalité exige qu'il endosse tel point de vue théorique plutôt que tel autre, alors la chose vaut a fortiori pour tout le monde. Car il ne saurait y avoir plusieurs rationalités différentes, et si un savant peut avoir raison de décider qu'une explication est à tous égards à ses yeux meilleure qu'une autre, il doit immanquablement en aller de même pour tous les autres chercheurs, et aussi pour la multitude. Car, en supposant que tous refassent le même cheminement réflexif et analytique, il est supposé que tous en arriveraient forcément et résolument à la même conclusion. C'est précisément avec cette conception individualiste et solipsiste de la pensée scientifique, oủ le fait que les scientifiques œuvrent en groupe ne change rien d'essentiel au processus du développement de la pensée, que Kuhn veut rompre définitivement. Mais, selon moi, cette rupture ne se fait absolument pas au risque de sacrifier la rationalité, comme on l'a très souvent reproché à Kuhn : elle se fait cependant au prix d'une redéfinition dans une perspective « collectiviste » de ce qui doit compter comme rationnel. Kuhn n'a jamais prétendu que les scientifiques n'avaient pas de jugement personnel et qu'ils faisaient montre d'un comportement de foule, chacun adoptant pour lui-même la ligne de conduite de la majorité ( $«$ mob rule »). Au contraire, Kuhn a insisté pour dire que dans un groupe de scientifiques, chacun a droit de divergence à l'intérieur de limites fixées par ce qui définit le groupe comme groupe partageant un langage, une problématique, une perspective et des objectifs de recherche communs. Le fait est que, malgre qu'ils puissent partager entre eux un même ensemble de critères de jugement, les scientifiques ne suivent pas de règles fixes dans l'application de tels critères. En conséquence, ils peuvent en venir à des conclusions différentes, voire fort divergentes. Ce qui a fait croire à certains critiques de Kuhn qu'il ouvrait irrémédiablement la porte à l'irrationalisme, c'est que celui-ci ait pu prétendre que le système des critères de jugement suivis par les scientifiques fonctionnait comme un système de valeurs et non pas comme un système d'opérateurs mécaniques, et que pour deux individus donnés, ce même système de valeurs sous-déterminait la solution qui pouvait apparaître à chacun comme étant la plus rationnelle. En effet, l'application de ces valeurs ou de ces critères de jugement pouvait très bien, suivant Kuhn, donner lieu à des choix non seulement divergents mais même incompatibles et contradictoires, sans quaucun de ces choix ne puisse être qualifié de meilleur parmi les choix effectués et aussi sans quaucun de ces choix ne puisse être déclaré « irrationnel » en lui-même.

Puisque, selon Kuhn, le jugement scientifique peut varier d'un individu chercheur à l'autre, le choix d'un cadre théorique au détriment 
d'un autre peut paraitre aux yeux de certains totalement personnel, c'est-à-dire enraciné dans des facteurs subjectifs de type esthétique ou métaphysique, et marqué au coin des aléas biographiques les plus contingents et les plus fantaisistes. Ce choix théorique n'étant plus unique et universalisable, il a pu sembler arbitraire et sans fondement rationnel $^{27}$. Pourtant, loin d'ouvrir la porte à l'arbitraire, comme si les jugements scientifiques ne s'enracinaient pas d'abord et avant tout dans des échanges d'arguments rationnels, Kuhn insiste même pour dire qu'en dépit du fait que les scientifiques ne partagent pas forcément la même opinion quant à savoir quelle théorie doit être considérée comme préférable, « le comportement scientifique est, lorsqu'envisagé globalement, le meilleur modèle de la rationalitè dont nous disposions $»^{28}$. Même dans des cas de figure exemplaires, cas particulièrement bien illustrés par l'entêtement obstiné d'un Priestley, un exemple sur lequel Kuhn revient à plusieurs occasions pour illustrer son propos, l'opiniâtreté scientifique, qui se nourrit d'arguments et n'a rien d'un caprice, n'est jamais un indice d'irrationalité. Et même lorsqu'aux yeux de ceux qui épousent un point de vue radicalement différent, la personne qui se bute, s'acharne et persévère peut être vue comme déraisonnable parce qu'à cours d'arguments, le comportement du scientifique qui résiste au changement n'est pas pour autant illogique suivant Kuhn. Car, si ce genre de disputes fondamentales entre scientifiques était affaire de preuves ou de démonstrations logico-mathématiques, et si l'on pouvait établir que la personne qui se refuse à changer d'idée le fait en écartant des arguments que, de l'intérieur de son système d'explication, elle devrait formellement admettre, il y aurait sans doute matière à soutenir que la résistance au changement est synonyme d'irrationalité ou de comportement illogique. Mais puisque les scientifiques se situant de part et d'autre de la coupure qui marquera le début d'une révolution ne disposent justement pas de telles preuves, Kuhn n'est pas enclin à qualifier le comportement des plus conservateurs d'illogique ou d'irrationnel, bien qu'il puisse s'agir, par ailleurs, d'un comportement déraisonnable parce qu'excessif. Pour Kuhn, même dans le cas où

27. Sur toute cette question des « raisons qui se cachent derrière le choix d'une théorie ", voir Paul Hoyningen-Huene, op. cit., p. 239-245. Cette analyse fait bien comprendre pourquoi Kuhn a soutenu que « ce problème du choix de paradigne ne peut jamais être réglé sans équivoque par le seul jeu de la logique et l'expérimentation » (SRS, p. 136).

28. Cf. « Notes on Lakatos » (dans R.C. Buck et R.S. Cohen, eds., PSA 1970. In Memory of Rudolf Camap. Dordrecht, Reidel, Boston Studies in the Philosophy of Science, vol. 8 . 1970, p. 137-146), p. I44. 
un scientifique se fait jusqu'au-boutiste et refuse à tout prix de changer de convictions théoriques, il n'existe aucun point au delà duquel l'on pourrait décréter qu'il devient pour ce scientifique logiquement absurde ou incohérent, et donc manifestement anti-scientifique, de se camper sur ses positions ${ }^{29}$.

On n'a pas bien vu que, loin de nier la rationalité du processus d'engagement en faveur d'un cadre théorique de recherche, Kuhn, en fait, l'établissait autrement. En effet, dans la perspective qui est la sienne, cette rationalité n'est plus d'abord ou seulement individuelle, car elle en est une de communauté de chercheurs. Bien que Kuhn insiste pour dire que ce processus de choix entre théories rivales n'est pas analysable en termes de « décision rationnelle individuelle », l'argument de Kuhn peut être néanmoins vu comme inspiré de la théorie de la décision rationnelle : tout se passe, en effet, comme si, à la faveur de jugements de valeur variant d'un chercheur à l'autre, les chercheurs répartissaient les risques entre eux de manière à ce que tous ne se trouvent jamais confrontés à un cul-de-sac en même temps. La rationalité scientifique dont parle Kuhn est donc une rationalité de groupe, ou, si l'on préfère, une rationalité évolutionnaire, étant entendu que ce qu'un regroupement scientifique d'individus cherche à faire, c'est résoudre une série de problèmes et ouvrir de nouvelles perspectives de recherche prometteuses. Ce que les premiers critiques de Kuhn n'ont pas bien jaugé, c'est à quel point une telle stratégie de recherche, basée sur la diversité des personnalités des chercheurs, aboutit finalement à une meilleure répartition des risques entre tous les individus, et donc constitue la meilleure stratégie de découverte à long terme, étant entendu que nul ne sait par avance quelle solution s'avérera la plus adéquate

29. La traductrice de SRS fait dire à Kuhn : « Bien que l'historien puisse toujours trouver des hommes - Priestley par exemple - qui ont manqué de raison en résistant aussi longtemps qu'ils l'ont fait, il ne découvrira pas un point oủ la résistance deviendrait logique ou antiscientifique » (p. 218). Il faut évidemment lire ici « illogique ». La même erreur a également èté faite dans la première traduction (p.r8g) mais n'a pas été relevée par Claude Savary. Se fondant en particulier sur ce passage, Hoyningen-Huene écrit : « This passage provides a good example of just how negligently Kuhn has often been read. We find Laudan asserting in his 1984 (il s'agit de Science and Values. The Aims of Science and Their Role in Scientific Debate, Berkeley, University of California Press. R.N.I, p. 72-73 that Kuhn claimed that it was perfectly reasonable for Priestley to insist on the phlogiston theory 》 (op. cit., p. 244, п. 124). Tout compte fait, il conviendrait plutôt de dire que pour Kuhn, Priestley fut sans doute déraisonnable, mais il ne fut jamais illogique. L'erreur de Laudan est d'amalgamer le « raisonnable » et le « rationnel », ce que Kuhn n'est manifestement pas prêt à faire. 
pour la résolution des problèmes dejà formulés mais aussi la plus profitable à la poursuite indéfinie de la recherche. Loin, donc, de renier la rationalité du travail scientifique, Kuhn se trouve plutôt à l'asseoir sur de nouvelles bases, et sur des fondements peut-être plus solides que les anciens. Rompant avec l'individualisme solipsiste de l'épistémologie et de la philosophie des sciences traditionnelle, Kuhn assigne à l'individu chercheur une nouvelle place en faisant voir que le comportement personnel du chercheur est en quelque sorte indissociable de celui du groupe auquel il appartient ou de la communauté scientifique dont il se réclame, institution hors de laquelle il ne pourrait mème plus songer à œuvrer comme un scientifique, faute de l'obligatoire soutien et de l'indispensable reconnaissance de ses pairs.

Il est indéniable que l'argumentation développée par Kuhn se voulait très critique des conceptions épistémologiques allant de pair avec la philosophie des sciences d'obédience logico-positiviste. Il serait injuste de refuser de reconnaître que les efforts de Kuhn, conjugués aux propres insuccès des penseurs de cette école ainsi qu'aux analyses d'autres philosophes extrêmement critiques des thèses logico-empiristes, ont atteint leur cible. Peu importe finalement qui est historiquement responsable de cette mort, l'empirisme logique est bel et bien mort, et Kuhn n'y est sans doute pas pour rien. Mais, à mon sens, il n'y a pas que cette doctrine philosophique particulière qui ait été atteinte par les arguments de Kuhn et de ses épigones : en fait. c'est toute la philosophie des sciences dans son ensemble qui s'est trouvée remise en cause, me semble-t-il, l'entreprise paraissant ne plus avoir de raison d'être. C'est, du moins, la lecture quej'entends maintenant proposer de cet épisode relativement récent de l'histoire de la philosophie des sciences. J aimerais faire voir qu'en accomplissant sa révolution, Kuhn a commis l'erreur stratégique de pousser la philosophie des sciences du côté de l'histoire et de la psychosociologie des sciences.

\section{3. llistaire, psychosociologie ou phillosophie des sciencees?}

Certes, je m'en voudrais de laisser croire que j' accepte comme allant de soi tous les arguments de Kuhn sur la détermination de l'observable par le théorique, ou encore ceux portant sur l'influence des facteurs idiosyncrasiques dans le choix entre théories rivales, ou enfin ceux censés montrer le peu d'importance de la réfutation d'une théorie dans le processus qui amène une communauté scientifique à changer de perspective de recherche. Cependant, que Kuhn rejette l'empirisme naïf de ceux qui croient en l'existence d'un langage observationnel neutre et universel, qu'il réinterprète la question des critères de choix théorique en termes de valeurs plutôt que de règles, et qu'il rejette le 
réfutationnisme poppérien sous prétexte qu'il s'agit là davantage d'une idéologie que d'une méthodologie en bonne et due forme ${ }^{30}$, rien dans tout cela ne me paraît injustifiable en principe. Toute la question est de savoir si de telles conclusions sont bien établies, et c'est le rôle des épistémologues et des philosophes des sciences d'en débattre. Néanmoins, je ne voudrais pas laisser entendre qu'en apportant ainsi de nouvelles réponses à des questions qui, par tradition, relevaient de la philosophie des sciences du vingtième siècle, Kuhn a tout simplement renouvelé l'ordre du jour de cette discipline. Il n'est pas évident que l'analyse des composantes et du fonctionnement de la rationalité collective des chercheurs scientifiques œuvrant en groupe relève de l'épistémologie et de la philosophie des sciences. Car, quoi qu'il en soit de la valeur de ce que Kuhn a appelé son « nouveau paradigme épistémologique anticartésien $»^{3^{\mathrm{I}}}$, le fond du problème n'est pas là. Certes, comme il est facile de le constater, l'œuvre de Kuhn continue de soulever des questions qui semblent exiger un traitement épistémologique ou encore un traitement logico-méthodologique. Mais l'œuvre de Kuhn pointe fondamentalement dans une tout autre direction : elle préside au détournement de l'entreprise philosophique vers un questionnement d'un autre ordre davantage inspiré de l'histoire des sciences, de la sociologie des sciences et de la psychologie cognitive. Kuhn nous propose en effet un chambardement radical qui devrait nous amener à substituer l'approche psychosociologique à l'approche logique de la science : les questions traditionnelles de la philosophie des sciences sont maintenant devenues des questions relevant de ce que Kuhn appelle en 1977 les « Metahistorical Studies ${ }^{32}$.

Deux options nouvelles - que Kuhn fait explicitement siennes me semblent à la base du « gestalt switch» que Kuhn a voulu provoquer

30. "Logic of Discovery or Psychology of Research ? », d'abord publié dans Criticism and the Growth of Knowledge (Imre Lakatos et Alan Musgrave, eds., Londres : Cambridge University Press, 1970, p. I-23), puis repris dans La Tension essentielle, op. cit., chap. XI. P. 356-390.

31. Kuhn n'a jamais présenté systématiquement sa nouvelle théorie de la connaissance, et il l'a plutôt fait par bribes. $C f$. entre autres son « Second Thoughts on Paradigms », traduit en français sous le titre « En repensant aux paradigmes » (dans La tension essentielle, op. cit., chap. xrI, p. 391-423, et spécialement p. 410 et sq.).

32. Les quatorze articles de Ia Tension essentielle sont répartis sous deux rubriques distinctes : « Historiographic Studies » et « Metahistorical Studies ». La traduction française (cf. op. cit.) distingue de son côté entre les « ètudes historiographiques » et les « études métahistoriques ». Toutes les questions que l'on pourrait prétendre de nature épistémologique ou logico-méthodologique sont regroupées dans la seconde partie du recueil. 
dans la philosophie des sciences ${ }^{33}$. Elles sont constitutives de la nouvelle tradition de recherche que Kuhn appelle de tous ses vœux pour déloger la philosophie des sciences traditionnelle de la place qu'elle occupe indûment selon lui. Mais ces deux options me paraissent absolument contestables et j'aimerais maintenant faire apercevoir qu'elles engendrent des difficultés insurmontables de l'intérieur même du prétendu paradigme révolutionnaire de Kuhn.

J'appelle la première option kuhnienne «loption psychosociologique». D'une certaine manière, nous nous retrouvons ici en terrain connu, car, en un sens, la thèse défendue par Kuhn est un avatar du psychologisme, contre lequel la logique et la philosophie de la connaissance scientifique eurent à lutter au début de ce siècle pour établir la légitimité intellectuelle de leur perspective théorique. A mon sens, c'est en quelque sorte le même débat qui se poursuit. C'est, en effet, à cette tradition philosophique davantage issue de Kant que de Descartes, que Kuhn me paraît s'en prendre ${ }^{34}$. Cette tradition anti-psychologiste est en effet assise sur une célèbre distinction de Kant, distinction incontournable entre la quaestio facti et la quaestio juris, c'est-à-dire, pour reprendre à mon compte la formulation kantienne de cette distinction, entre la question de la « dérivation psychologique » des concepts dans la conscience

33. «Gestalt switch» est l'expression dont Kuhn se sert dès 1962 dans SRS pour expliciter son concept de « révolution scientifique ». Il n'est donc pas sans intérêt de noter que, dans « Logique de la découverte ou psychologie de la recherche? ? (loc.cit.), texte publié pour la première fois en 1970 mais lu en 1965 au colloque de Bedford College où s'affrontèrent Kuhn et Popper (cf. Criticism and the Growth of Knowledge. op.cit.), Kuhn, se faisant insistant sur ce qui le sépare de Popper, termine son texte, à l'occasion de remarques portant sur la fonction de ce que Popper considère être des « règles méthodologiques », en utilisant cette même notion de « gestalt switch »: « Qu'il (Popper) ne les (les règles méthodologiques) voie jamais, à ce que je pense, comme des impératifs sociopsychologiques, ce qu'ils sont en fait, est une preuve supplémentaire du basculement gestaltien qui nous sépare encore grandement 》 (cf. La Tension essentielle, op. cit., p. 39ol. L'ensemble du texte fait bien voir qu'il n'y a pas que la philosophie rationaliste critique de Popper qui soit remise en question par Kuhn mais bien l'entreprise de la philosophie des sciences elle-même en tant qu'elle se veut une méthodologie de la connaissance scientifique.

34. Je ne doute pas le moins du monde que, comme le fait voir Paul HoyningenHuene dans une argumentation audacieuse et au demeurant fort convaincante, la doctrine kuhnienne puisse recevoir un éclairage tout à fait intéressant en étant interprétée dans la perspective de l'idéalisme kantien (cf. « Epilogue. Reality as Understood by Kuhn's Philosophy of Science », op. cit., p. 267-27I). Mais il n'en reste pas moins que toute l'entreprise de Kuhn, du fait justement de son orientation résolument historicisante et psychosociologisante, se situe selon moi à mille lieues d'une philosophie de type transcendantal. 
subjective de chacun et la question de leur «prétention à la validité » universelle. C'est la même optique qui se trouve défendue chez Frege à propos des mathematiques. Selon Frege, en effet, il importe à tout prix de distinguer entre la description de l'origine d'une idée ou d'une proposition, et la preuve de cette proposition. L'anti-psychologisme est à ce point fondamental qu' on le retrouve partout et même par-delà les frontières séparant des systèmes épistémologiques aussi divergents que ceux de Carnap et de Popper. Et j'aimerais rappeler que la distinction, dont la formulation canonique est due à Hans Reichenbach ${ }^{35}$, entre contexte de découverte et contexte de justification n'est qu'une reformulation de cette distinction kantienne. La chose apparaît clairement dans la présentation qu'en fait, entre autres, Herbert Feigl dans sa carac térisation du programme de recherche néo-positiviste ${ }^{36}$. Or cette distinction cruciale, voire capitale, que je n'ai, pour ma part, aucune difficulté à accepter, est rejetée par Kuhn de façon explicite ${ }^{37}$. Je ne suis pas certain que cette distinction soit toujours bien comprise par Kuhn,

35. On dit habituellement que c'est Reichenbach qui a formulé cette distinction (cf. $\mathrm{H}$. Reichenbach, Experience and Prediction. An Analysis of the Foundations and the Structure of Knowledge. Chicago, The University of Chicago Press, 1938; cf. aussi L'Avenement de la philosophie scientifique, Paris, Flammarion, deuxième partie, chap. $8:$ : La connaissance, instrument de prévision ou connaissance prédictionnelle », p 197214. particulièrement aux pages I98-199, oủ l'on notera la grossière erreur de traduction qui introduit un contresens. Mais, même si elle ne se trouve pas formulée dans les termes qu'utilisera Reichenbach, cette même distinction se trouve déjà faite par Popper dès 1934 ( $c f$. La Logique de la découverte scientifique, Paris, Payot, I973. chap. I, section 2, p. 27-28). Et Popper fait explicitement le lien avec Kant sur lequel j'insiste moi-même ici.

36. « Our concern was not with the psychological origins or the social conditions of the cognitive enterprise. Our distinction was based on differences in the method of validation. In Reichenbach's terminology, we were analysing knowledge claims in the "context of justification" and not in the "context of discovery". We always admitted that all sorts of intuitive processes (e.g., "hunches", consciously or subconsciously discerned analogies), may well be extremely instrumental (heuristically) in the genesis of hypotheses and theories. We never claimed that great scientific theories could be constructed by a logic-machine. (This was long before the age of computers - and who knows what may get be achieved by them ?) We firmly held (with Einstein, and with Popper) that there is no straight logical path that leads from the data of observation to an explanatory theory ». Cf. « Wiener Kreis in America 》 (Perspectives in American History, 2, 1968, p. 630-75), p. 653.

37. Kuhn critique cette distinction d'entrée de jeu : $c f$. SRS p. 26-27. Il y revient dans « Objectivité, jugement de valeur et choix d'une théorie » (loc. cit., p. 432-436). Sur cette question, la littérature est fort abondante. Mais la meilleure analyse récente est à mon avis due à Paul Hoyningen-Huene (cf. Reconstructing Scientific Revolutions, op.cit., p. $245^{-25}$ ). 
pas plus que je ne suis certain qu'il y ait possibilité de la formuler logiquement de manière à ce qu'elle fasse consensus. Cependant, il est peut-être possible de la clarifier davantage.

Etablissons d'abord pour quels motifs Kuhn rejette explicitement cette distinction. Il le fait d'une manière qui paraîtra constamment irrecevable à celui qui admet la distinction. Par exemple, dans « Objectivité, jugement de valeur et choix d'une théorie ». Kuhn est amené à rejeter la distinction à la faveur d'une critique adressée à Israël Scheffler ${ }^{38}$. Selon Scheffler, les facteurs subjectifs identifiés par Kuhn comme jouant le rôle décisif dans les épisodes au cours desquels les scientifiques choisissent entre théories rivales sont en fait des facteurs qui relèvent du contexte de découverte mais non du contexte de justification. Ces facteurs jouent, selon Scheffler, au plan de la genèse psychologique des idées, qui est personnelle à chacun, mais non au plan de ce qui fait qu'un argument est bien fondé ou non, et les raisons qu'on pourrait alléguer en l'occurrence ne sauraient être qu'universellement admissibles. Or, comme on le verra, il est très clair que, dans le passage incriminé, Kuhn confond deux questions, à savoir, d'une part, celle de savoir ce qui peut logiquement compter comme une justification de la théorie elle-même, et, d'autre part, celle de savoir ce qui justifie le chercheur d'opter pour une théorie particulière plutôt que pour une autre. Si la seconde question est certainement susceptible d'une analyse psychosociologique, la première ne saurait l'être qu'au prix d'une erreur de catégorisation.

Mais parce que Kuhn ne semble pas reconnaître lajustesse de cette distinction entre catégories de questions, il va plus loin encore dans l'erreur de raisonnement. En effet, non seulement ne distingue-t-il pas entre question de droit et question de fait, mais encore prétend-il tenir simultanément un discours descriptif et un discours normatif. Car Kuhn entend non seulement analyser ce qu'il en est de l'activité scientifique telle qu'elle a cours dans l'histoire, mais il pretend egalement prescrire aux chercheurs, en conformité avec les résultats mis en évidence dans son analyse empirique de l'histoire des sciences, un comportement qui soit scientifiquement normatif parce que supérieur à celui que prescrivent des épistémologues ou des méthodologues comme Popper.

38. Scheffler insiste pour dire qu'avec la mise en question de la standard view. notamment par Kuhn, « the very distinction between justification and discovery breaks down as well » (Science and Subjectivity, Indianapolis, New York, Kansas City : The Bobbs-Merrill Co., Inc., 1967, P. 17). Kuhn fait explicitement référence à cette critique mais son analyse me paraît passer complètement à côté de la question. 
Kuhn nous dit en effet :

Si j'ai par devers moi une théorie expliquant comment et pourquoi la science fonctionne comme elle le fait, cela doit nécessairement avoir des implications pour ce qui concerne la façon dont les scientifiques devraient se comporter si leur entreprise se veut florissante. La structure de mon argument est simple et, je pense, inattaquable : les scientifiques se comportent de telle et telle façons; ces modes de comportement manifestent (et ici la théorie entre en jeu) telle et telle fonctions essentielles; en l'absence d'un mode différent qui puisse servir des fonctions similaires, les scientifiques devraient continuer de se comporter essentiellement comme ils le font déjà si leur objectif est d'améliorer la connaissance scientifique ${ }^{39}$.

Formulé comme il l'est, cet argument de Kuhn est une simple tautologie : à défaut de pouvoir faire mieux, il faut continuer de faire comme on fait déjà si l'on poursuit toujours les mêmes objectifs. Rien n'indique cependant que l'on ne puisse faire mieux : et c'est précisément le contraire que présuppose toute réflexion philosophique de nature logico-méthodologique. Mais si ce raisonnement prescriptif n'est pas logiquement vide, alors Kuhn me parait clairement tenter de dériver une prescription à partir d'une description empirique de la situation dans laquelle se trouvent les scientifiques, les plus performants comme les moins clairvoyants. Ce problème est un problème logique et aucune analyse historique ou psychosociologique ne parviendra à le résoudre. Kuhn fonde, en effet, ce qui deuraitêtre fait par les scientifiques sur ce qu'ils ont faitjusqu'ici, et dans certains cas avec un certain succès. Le sociologisme de Kuhn est marqué tout autant au coin de ce que Salmon appelle la «genetic fallacy ${ }^{40}$ que de ce que, depuis Hume, on considère être la « is-ought fallacy». Mais l'abandon du programme envisageant la philosophie des sciences comme une « logique méthodologique de la connaissance scientifique » au profit d'un programme prétendument révolutionnaire qui l'envisage plutôt comme une « sociologie des communautés scientifiques ${ }^{4 \mathrm{~T}}$ empêche à toutes fins utiles que ce débat philosophique fondamental soit mené dans la perspective adéquate et à l'aide des instruments appropriés, c'est-à-dire à

39. Cf. « Refflections on my Critics », loc. cit., p. 237.

40. W.C. Salmon, « Bayes's Theorem and the History of Science », Minnesota Studies in the Philosophy of Science V, 1970, p. 68-86.

41. Dans « Reflections on my Critics », Kuhn affirme préférer s'identifier à la sociologie plutôt qu'à la «psychologie sociale » (p. 240). On aurait pu croire pourtant qu'il défendait le point de vue de la psychology of research si l'on s'en remet au titre de sa conférence au Colloque de Bedford College en 1965. 
l'aide de l'analyse logique des concepts. Car si le « gestalt switch» souhaité par Kuhn réussit, nous nous retrouvons d'emblée en histoire et en psychosociologie des sciences, et il n'y a plus aucun moyen de résoudre le problème posé, qui est d'ordre logique et méthodologique. Voilà une première raison qui m'amène à soutenir que l'entreprise de Kuhn, parce qu'elle a manifestement induit un virage historiciste et psychosociologiste, a amplifié la crise de la philosophie des sciences post-positiviste plutôt que de contribuer à la résorber ${ }^{42}$. L'analyse logique et méthodologique est maintenant devenue illégitime aux yeux de plusieurs qui se reclament ouvertement de Kuhn et qui se refusent tout simplement à se placer de ce point de vue.

Jappelle la seconde option favorisée par Kuhn « l'option métahistoriographique » et l'examen de cette seconde option kuhnienne me fournira une seconde raison, allant de pair avec la première, de penser que l'approche kuhnienne de la science a contribué à sa façon à prolonger la crise de la philosophie des sciences de l'ère post-positiviste. Il convient de rappeler que l'objectif de la philosophie des sciences jusqu'à Kuhn a été, d'une part, de procéder à la reconstruction rationnelle des théories scientifiques particulières mais, d'autre part et plus généralement, de contribuer à l'élucidation de la nature et du fonctionnement logico-méthodologique du savoir scientifique. Au cœur de ce programme de recherche a pris place, pendant longtemps, la question de savoir s'il était possible de définir rigoureusement un critère qui caractériserait distinctement la connaissance scientifique telle qu'on la trouve à l'œuvre dans les diverses disciplines empiriques. L'abandon du critère de vérifiabilité n'a pas marqué, comme on le sait, la fin de cette recherche, qui a débouché sur une théorie de la confirmation et de la testabilité (Carnap) ou sur une théorie de la réfutabilité et de la corroboration (Popper). Il convient d'affirmer aujourd'hui que l'échec de la doctrine logico-positiviste de la « signification empirique » des termes théoriques ne signifie pas qu'il faille abandonner à tout jamais toute

42. On peut pretendre que cette crise se résorbe maintenant parce qu'une ligne de partage de plus en plus étanche sépare aujourd'hui les eaux dans lesquelles frayent les philosophes des sciences, qui sont retournés à leurs préoccupations logico-méthodologiques traditionnelles, de celles sur lesquelles voguent allègrement les sociologues des sciences, dont la majorité semble avoir endossé dogmatiquement le « programme fort » de David Bloor et Barry Barnes (cf. David Bloor, Knowledge and Social Imagery. $2^{\mathrm{e}}$ ed., Chicago et Londres, The University of Chicago Press, I9gI; voir en particulier son "Afterword : Attacks on the Strong Programme », p. 163-85). Sur cette question, le recueil édité par J.R. Brown constitue encore le meilleur ouvrage de référence (cf. Scientific Rationality : The Sociological Tum, Dordrecht, D. Reidel, Ig84). 
réflexion de cette nature, pas plus que les difficultés mises au jour dans la théorie de la vérisimilarité ne doivent entraîner l'abandon de tout projet d'analyse logique du rapport de la connaissance scientifique à la vérité.

La question de savoir ce qu'est une théorie authentique de la science empirique est encore ouverte, peu importe que l'on parvienne ou non à caractériser de façon satisfaisante la nature du savoir scientifique à l'aide d'un critère de signification ou à l'aide d'un critère de démarcation. Bref, la notion de scientificité elle-même doit rester susceptible d'analyse logique, méthodologique et épistémologique. Or il arrive que Kuhn, dès son ouvrage de ig62, nous incite à renoncer à cette sorte de philosophie des sciences ${ }^{43}$ pour la seule et unique raison que, selon lui, le praticien des sciences se moque éperdument de la « définition » que le philosophe est susceptible de donner du terme «science ». La logique de la science développée par les méthodologues ne semble guère intéresser les scientifiques, si l'on se fie du moins à ce qu'en dit Kuhn, car, apparemment, ils n'en ont que faire. Pourtant, l'épistémologie et la méthodologie contemporaines n'envisagent pas le concept de « science » comme un concept clair et défini : c'est plutôt une notion problématique qui a besoin d'analyse et de clarification. Cependant, la philosophie des sciences contemporaine accepte tout à fait de considérer la science elle-même comme un fait au sens où Kant considérait la science newtonienne comme un acquis incontestable dont il fallait tenter de comprendre les conditions de possibilitè. Par contre, le « métahistorien des sciences », qui s'assigne la tâche d'analyser la formation et la transformation des matrices disciplinaires particulières ainsi que la tâche plus générale de fournir une explication psycho- et sociohistorique du développement des programmes et des traditions de recherche, rejette d'emblée comme non pertinentes toutes les questions épistémologiques et logico-méthodologiques afférentes au concept de science. Des questions comme les suivantes : «Existe-t-il un critère de scientificité démarquant les sciences authentiques des pseudo-sciences? », « Comment convient-il de caractériser les structures logiques des théories scientifiques idéales? 》, «Peut-on formuler des règles méthodologiques incontournables en science empirique? », «Existe-t-il une différence entre les savoirs purement formels et les savoirs empiriques?», «Les idéalités mathématiques correspondentelles à quelque chose de réel ? », « Y a-t-il une différence de nature ou seulement une différence de degré entre les sciences naturelles et les 
sciences sociales ?», « Comment peut-on caractériser la notion de testabilité ?», «Qu'est-ce qu'une bonne explication scientifique ?», «Qu'est-ce qui rend une preuve empirique scientifiquement acceptable? ». De telles questions risquent donc d'être jugées dorénavant irrecevables. Car n’étant ni d’ordre métahistoriographique, ni d'orientation psychosociologique, ces questions sont, suivant Kuhn, sans importance pour les scientifiques eux-mêmes. Et puisqu'elles sont insignifiantes pour les scientifiques pratiquant la « science normale», on devrait les abandonner à leur sort et ne plus s'en préoccuper.

Les épigones de Kuhn diront peut-être non pas qu'elles sont irrecevables mais que l'apport de Kuhn est d'avoir ouvert la voie au traitement historique et sociologique de ces questions : d'en avoir fait des questions métahistoriographiques. Mais il y a ici, manifestement, confusion. Car dire cela, c'est croire, par exemple, qu'une théorie empirique de l'empiricité est possible. Un tel point de vue repose en fait sur une pétition de principe : car vouloir caractériser scientifiquement ce qu'il en est de la scientificité, ou vouloir établir empiriquement ce qu'il en est de l'empiricité, c'est tenir pour non problématique ce qu'il s'agit justement de clarifier par la voie de l'analyse philosophique. C'est pourquoi un deuxième motif de crise s'est en quelque sorte subrepticement installé au cour de la philosophie des sciences d'aujourd'hui. Loin que l'on propose à la philosophie des sciences de poursuivre des débats amorcés dans le sillage du conventionnalisme français, du pragmatisme américain, du positivisme logique et du falsificationnisme, le changement de garde favorisé par Kuhn en a amené plusieurs à troquer polémiquement la philosophie des sciences pour une histoire psychosociologique des sciences ${ }^{44}$.

Dans cette nouvelle perspective, la logique et la méthodologie cèdent en effet la place à l'histoire et à la sociologie. De la sorte, des questions à mon avis importantes, voire cruciales, perdent jusqu'à leur légitimité philosophique. Tous les concepts comme ceux de vérité ou d'« approximation de la vérité », de preuve empirique, de confirmation, de test, d'observation, de théorie, de prédiction, d'explication, de loi, etc., qui ne sont pas analysables autrement que logiquement et méthodologiquement, se trouvent au mieux réduits à des notions dont il s'agit seulement de retracer la genèse et la transformation sociohistorique, et au pire laissés pour compte. C'est du moins ce qui risquerait

44. A mon avis, toute la sociologie des sciences post-mertonienne se réclamant du «programme fort », peu importent les nuances théoriques plus ou moins subtiles qu'opère chaque école de pensée, prête le flanc à cette critique. 
de se produire si l'on s'en remettait à $\mathrm{Kuhn}^{45}$. Car, suivant Kuhn, toutes ces notions sont à chaque fois différemment redéfinies, implicitement ou explicitement, à l'intérieur de chaque paradigme. Il n'y a donc pas lieu de supporter le programme de recherche d'une sorte de philosophia perennis qui envisagerait encore aujourd'hui ces notions métascientifiques comme s'il s'agissait de notions intemporelles ou anhistoriques, ou encore, ce qui revient strictement au même pour Kuhn, comme si on pouvait croire ces notions susceptibles d'une élucidation purement logico-méthodologique.

La conséquence immédiate de la mise en marche du programme de recherche kuhnien, s'il était suivi à la lettre, serait d'annuler toute philosophie des sciences en lui déniant toute utilité. En effet, dans la perspective de Kuhn, l'épistémologie et la méthodologie, au sens traditionnel de ces termes, n'ont tout simplement plus de légitimité. Ces disciplines doivent être remplacées par un programme de recherche tenu pour « révolutionnaire » et exclusivement base sur la sociologie et l'histoire des sciences. La philosophie des sciences de l'ère pré-kuhnienne, qui avait la velléité de procéder à la critique épistémologique de la connaissance scientifique et à la critique logico-méthodologique des théories scientifiques - y compris, du reste, celles de la sociologie et de l'histoire des sciences -, n'a apparemment plus de raison d'être si l'argumentation de Kuhn est fondée. En effet, si Kuhn devait finir par faire prévaloir sa perspective de recherche, il s'ensuivrait un abandon des modes d'analyse propres à l'épistémologie, à la logique et à la méthodologie des sciences. S'il devait avoir lieu, cet abandon aurait ceci de particulièrement pervers qu'il empêcherait que certaines questions éminemment philosophiques soulevées par l'analyse kuhnienne de la science soient encore traitées comme telles aujourd'hui et continuent de paraître intellectuellement admissibles.

Je conclurai en affirmant que si elle veut continuer d'être une réflexion de type épistémologique et méthodologique, la philosophie des sciences doit redécouvrir les vertus de l'analyse logique et de l'analyse conceptuelle. Loin de rompre avec les grandes traditions de la logique des sciences au vingtième siècle de Duhem à Popper et à Carnap, elle devrait s'y ressourcer à nouveau et surtout ne pas troquer son approche contre celle des historiens et des psychosociologues. Parce que ce troc a pu paraître acceptable et qu'il le semble encore aujourd'hui à plusieurs, il y a bien eu et il y a toujours une crise larvée dans la philosophie des sciences de cette fin de siècle. Et s'il y a bien une crise de

45. L'affirmation est explicite : $c f$. « Reflections on my Critics », loc. cit., p. 266. 
la philosophie des sciences après Kuhn, c'est d'abord et avant tout parce que le nouveau programme de recherche favorisè par Kuhn, lui qui s'est porté caution de ce virage historiciste et psychosociologiste, peut nous priver des moyens de traiter adéquatement de questions philosophiques incontournables, questions concernant la connaissance scientifique qui se posent de toute façon depuis longtemps, c'est-à-dire depuis au moins l'avènement de la science moderne. C'est pourquoi, même si cette crise est déjà peut-être derrière nous pour l'essentiel, elle mérite encore qu'on en médite les tenants et les aboutissants ${ }^{46}$.

Département de philosophie

Université du Québec à Montréal

46. Je remercie le Fonds FCAR du Québec ainsi que le Conseil de recherches en sciences humaines du Canada pour leur soutien financier. Je remercie également Gérald Lafleur de ses commentaires sur une version antérieure. 